\title{
Increased Paracrine Immunomodulatory Potential of Mesenchymal Stromal Cells in Three-Dimensional Culture
}

\author{
Bjarke Follin, MSc, Morten Juhl, MSc, Smadar Cohen, $\mathrm{PhD}$, Anders Elm Pedersen, MD, PhD, \\ Jens Kastrup, DMSc, MD, and Annette Ekblond, $\mathrm{PhD}^{1}$
}

Mesenchymal stromal/stem cells (MSCs) have been investigated extensively through the past years, proving to have great clinical therapeutic potential. In vitro cultivation of MSCs in three-dimensional (3D) culture systems, such as scaffolds, hydrogels, or spheroids, have recently gained attention for tissue engineering applications. Studies on MSC spheroids demonstrated that such cultivation increased the paracrine immunomodulatory potential of the MSCs, accompanied by phenotypic alterations. In this review, we gather results from recent experimental studies on the immunomodulatory abilities of MSCs when cultured as spheroids or in biomaterials like scaffolds or hydrogels compared to regular two-dimensional (2D) culture and show that alterations occurring to MSCs in spheroids also occur in MSCs in biomaterials. We provide a brief description of known mechanisms of MSC immunomodulatory capacity and how they are altered in the two 3D culture systems, together with phenotypic cellular changes. Based on the present knowledge, we highlight vital areas in need of further investigation. The impact of 3D environments on immunomodulation has great potential for tissue engineering and cellular therapy, and this is the first review to gather this knowledge with a comparison across different $3 \mathrm{D}$ environments.

\section{Introduction}

$\mathbf{C}$ ELL THERAPY BASED on mesenchymal stromal/stem cells (MSCs) is emerging as a clinical experimental treatment option for various diseases and this field of research is expanding rapidly. The immunomodulatory potential of MSCs is well documented, with several mechanisms elucidated for various targets in the immune system. However, the knowledge of MSC behavior and capabilities is primarily based on research in two-dimensional (2D) culture on tissue culture plastic (TCP) surfaces. ${ }^{1}$ There is evidence for change in immunomodulatory ability when MSCs are cultivated in three-dimensional (3D) spheroids compared to 2D culture, ${ }^{2-}$ 6 and lately, additional evidence points toward a similar change when MSCs are cultivated in biomaterials such as hydrogels and scaffolds. ${ }^{7-11}$ This review summarizes recent findings on the immunomodulatory mechanisms of MSCs cultivated as spheroids and in 3D biomaterial cultures com- pared to MSCs on 2D TCP surfaces, published within the past 5 years. We evaluate and discuss differences in MSC immunomodulatory capacity and phenotype between those cultured in spheroids and biomaterials.

\section{Mesenchymal Stromal/Stem Cells}

MSCs were originally characterized by their plastic adherence, pattern of phenotypical nonspecific surface markers, such as CD105, CD90, CD73, CD29, and CD166, and their inducible differentiation potential into osteogenic, chondrogenic, and adipogenic lineages. ${ }^{12,13}$ Cells matching these criteria have been found in most adult tissues, including bone marrow and adipose tissue. Although the potential has been shown to differ between MSCs from different tissue sources, MSCs fundamentally have the same set of abilities of secreting trophic, angiogenic, and immunomodulatory signals. ${ }^{14-16}$ The ability of immunomodulation is of special interest for this review.

\footnotetext{
${ }^{1}$ Cardiology Stem Cell Center, The Heart Center, Rigshospitalet, Copenhagen University Hospital, Copenhagen, Denmark.

${ }^{2}$ Regenerative Medicine and Stem Cell Research Center, the Avram and Stella Goldstein-Goren Department of Biotechnology and Engineering, Ben-Gurion University of the Negev, Beer Sheva, Israel.

${ }^{3}$ Department of Immunology and Microbiology, University of Copenhagen, Copenhagen, Denmark.

(C) Bjarke Follin et al. 2016; Published by Mary Ann Libert, Inc. This Open Access article is distributed under the terms of the Creative Commons Attribution Noncommercial License (http://creativecommons.org/licenses/by-nc/4.0/) which permits any noncommercial use, distribution, and reproduction in any medium, provided the original author(s) and the source are credited.
} 


\section{The Immunomodulatory Ability of MSCs}

The immunomodulatory abilities of MSCs have been described in depth in excellent reviews made by Le Blanc and Mougiakakos and Gebler et al., and we will only touch upon the mechanisms that are important for the understanding of this review. ${ }^{17,18}$

MSCs have several means by which they can affect the individual immune cell types. Overall, MSCs exercise their immunomodulatory effects by inhibiting proliferation and maturation of proinflammatory or effector immune cells, as well as driving certain immune cells toward tolerogenic and anti-inflammatory phenotypes. ${ }^{18} \mathrm{We}$ will briefly describe some mechanisms for the different immune cell types of interest for this review.

Macrophages consist of a heterogeneous population of polarized cells with widely varying effects in terms of microbicidal, wound healing, or regulatory activity. ${ }^{19}$ Several factors produced by MSCs have been described as having a direct influence on macrophage polarization and function. Prostaglandin E2 (PGE2), hepatocyte growth factor (HGF), and transforming growth factor beta (TGF- $\beta$ ) are able to suppress classical proinflammatory markers and shift macrophage polarization toward an anti-inflammatory phenotype. PGE2, a product of arachidonic acid metabolism by COX-2, is one of the major anti-inflammatory mediators in MSC immunomodulatory function. ${ }^{2}$ HGF is identified as one of the main factors secreted by MSCs contributing to wound healing. In addition to the trophic effects of HGF, it has also been found to suppress several immune cell types. ${ }^{2}$ The pleiotropic cytokine TGF- $\beta$ has important roles in initiation and resolution of inflammation, as well as induction of tolerance, through different types of targeted immune cells. ${ }^{20}$ The anti-inflammatory macrophage phenotype secretes Interleukin-10 (IL-10), which acts in an immunosuppressive manner on other immune cells such as lymphocytes. In vivo, this transition has also been shown to be the result of MSC therapy, with local anti-inflammatory macrophages residing in the tissue afterward, probably mediating some of the regenerative potential of the MSCs. ${ }^{21}$

The most potent antigen-presenting cell, the dendritic cell (DC), can be affected in the same way. Many MSC-secreted factors affecting macrophages have an overlapping inhibitory effect on DC maturation and function. This is true for PGE2, HGF, and TGF- $\beta$, and also TSG-6 and MCP- 1 . The combined effect skews the balance toward a more tolerogenic DC, displaying lower levels of major histocompatibility complexes, costimulatory signals, and effector cytokines. Tolerogenic DCs have suppressive effects on $\mathrm{T}$ cells, for example, by expression of indoleamine 2,3-dioxygenase $(I D O)$. IDO and the related tryptophan 2,3-dioxygenase are rate-limiting enzymes in the kynurenine pathway, and depletion of their substrate, the essential amino acid tryptophan, leads to immunosuppression not only by T-cell starvation but also by signaling through metabolites. ${ }^{22,23}$ In addition to the mentioned paracrine attenuation of DC maturation, several groups have found the DC maturation inhibition by the MSCs to be contact dependent, which suggests that there are contact mechanisms currently unknown working between DCs and MSCs. ${ }^{24,25}$

In case an initiation of lymphocyte response does occur, despite the suppression of antigen-presenting cells, the MSCs can inhibit the proliferation of lymphocytes. This is done through many of the same mechanisms used by the antiinflammatory subtypes of immune cells and includes the release of IDO and IL-10. ${ }^{26} \mathrm{HGF}$ has also been shown to be one of the central immunosuppressive cytokines for suppression of T-cell proliferation. ${ }^{27}$ These paracrine mechanisms work hand in hand with the inhibition through cell-cell interaction with intercellular adhesion molecule (ICAM/CD54) and Programmed Death Ligand-1 (PD-L1) on MSCs. ${ }^{28,29}$

In addition to inhibiting proliferation of lymphocytes, MSCs have been shown to be able to increase differentiation of $\mathrm{T}$ cells into regulatory $\mathrm{T}$ cells (Treg). Treg cells are generated directly by MSC secretion of IL-10, PGE2, and TGF- $\beta 1$ or indirectly by induced anti-inflammatory macrophages. ${ }^{26,30,31}$ Treg cells in turn secrete IL-10 and TGF- $\beta$, block the target cell by cell-cell contact, and competitively consume inflammatory growth factors. ${ }^{32}$

MSCs do not always secrete large quantities of immunomodulatory mediators. As an example, IDO is not detectable in nonstimulated MSC cultures. Instead, MSCs are activated by inflammatory signals, such as high levels of TNF- $\alpha$ and INF- $\gamma .{ }^{33}$ This shifts the MSCs toward an immunosuppressive phenotype, which secretes higher amounts of IDO, PGE2, IL10 , TGF- $\beta$, and HGF. ${ }^{34}$ These immunomodulatory abilities of the MSCs have all been observed in 2D cultures on TCP. Two-dimensional TCP culture is straightforward and yields numerous proliferative cells, which are easily characterized. ${ }^{12,13}$ However, the natural environment of MSCs is 3D and within a matrix, which is more complex and pliable compared to TCP. This means that our understanding of MSC function, for example, in terms of immunomodulation, is only an approximation of the actual in vivo function. ${ }^{1}$

\section{MSCs in 3D Culture}

For the remainder of the review, we will separate 3D cultures into two categories: spheroids and biomaterials. This is to distinguish between 3D environments made up from cells with those made up from biomaterials. They also represent two different incentives for 3D culture. The MSC spheroids were originally created to investigate in vivo behavior of administered MSCs, which spontaneously assembled into spheroids in the lungs of rats. ${ }^{3,35}$ Findings of spontaneous spheroid formation in vivo have not been reported on a regular basis since, but the promise of improved MSC ability spawned several subsequent in vitro studies. Simultaneously, a group used 3D bioreactors in an attempt to enhance MSC cell culture and found beneficial effects of the spheroid form. ${ }^{36}$ Since then, several different ways of assembling MSC spheroids have been exploited, the most widely used being hanging drops or nonadherent culture, which both result in cell clusters due to the lack of substrate adherence. ${ }^{35}$ Scaffolds and hydrogels are created from biomaterials for tissue engineering purposes with the aim to replace damaged tissue with cellseeded scaffolds. The scaffolds are meant to control the physical environment with the primary aim of differentiating cells toward a certain lineage, or investigating topographical cues in terms of guiding differentiation. Another application for this group is to use hydrogels to improve retention of administered cells during cell therapy. These constructs have primarily been investigated with regard to cell viability, construct implantability, and biocompatibility. 


\section{Evidence for Increased Immunomodulatory Abilities in 3D Cultures}

Most of the included immunomodulatory studies have avoided direct cell-cell contact by using transwell cocultures with MSCs or a conditioned medium from the MSCs on immune cells. Therefore, the results describe an isolated paracrine effect. The MSCs in all the included studies were undifferentiated, which is important to mention since differentiation of MSCs has been shown to decrease their immunosuppressive ability. ${ }^{37}$ However, since the subject of this review is the difference between $2 \mathrm{D}$ and $3 \mathrm{D}$, and the included studies all refer to a $2 \mathrm{D}$ control with the same cell type, we will not discuss this. In addition, the MSCs in all the studies were from healthy donors and used in passage $2-5$ for most studies and below passage 7 in one study. Reviewed comparisons between these immunosuppressive effects obtained in $3 \mathrm{D}$ versus $2 \mathrm{D}$ cultures for both spheroids and biomaterials in transwell cocultures are summarized in Table 1.

\section{Spheroids}

The enhanced immunosuppressive effects of MSCs in spheroids have been observed with macrophage secretion assays. Under normal circumstances in 2D TCP culture, MSCs decrease stimulated macrophage secretion of the inflammatory cytokine TNF- $\alpha$ in transwell coculture. This TNF- $\alpha$ suppressive effect can be enhanced if the MSCs are in spheroid form compared to 2D TCP culture, whether stimulated with lipopolysaccharide (LPS) or LPS in combination with IFN- $\gamma .{ }^{3,38}$ The TNF- $\alpha$ decrease in the LPS setup was later shown to be concurrent with macrophage changes, such as increase in both IL-10 secretion and CD206 expression, hallmarks of anti-inflammatory macrophages. IL-10 levels were higher in cocultures with MSC spheroid than in cocultures with MSCs in 2D, suggesting more anti-inflammatory macrophages. The same study found evidence for PGE2 being responsible for macrophage polarization and PGE2 levels to be higher in MSCs in spheroids than 2D. ${ }^{5}$

In addition to PGE2, HGF is increased in MSC spheroids compared to monolayers, ${ }^{3,5,6,39}$ which is also true for TSG-6. ${ }^{3,6}$ Among other functions, TSG- 6 works as a countermeasure to TNF- $\alpha$ and IL-1 inflammation by negative feedback loop and in particular by inhibiting neutrophil migration. $^{26,40}$ Increased levels of TSG-6 secretion from lung MSC microemboli spheroids were one of the first observations indicating that the MSC paracrine activity is higher in 3D than $2 \mathrm{D} .{ }^{3}$ Of the studies included in this review, only Zimmermann and McDevitt investigated IDO, and they found no difference in IDO between MSCs in spheroids and 2D culture. ${ }^{38}$ Since IDO is generally attributed to many of the immunomodulatory effects of MSCs, it would be interesting to investigate MSC expressions of IDO in various $3 \mathrm{D}$ environments.

\section{Biomaterials}

While macrophage secretion was investigated in the studies with spheroids, macrophage migration was mostly studied with biomaterials. Macrophage migration through a porous membrane toward the conditioned medium from crosslinked polystyrene scaffold MSC coculture was slower than toward the medium from monolayer coculture. ${ }^{7}$ In support of this migration assay, the conditioned medium from endothelial cells (ECs) in collagen scaffolds decreased

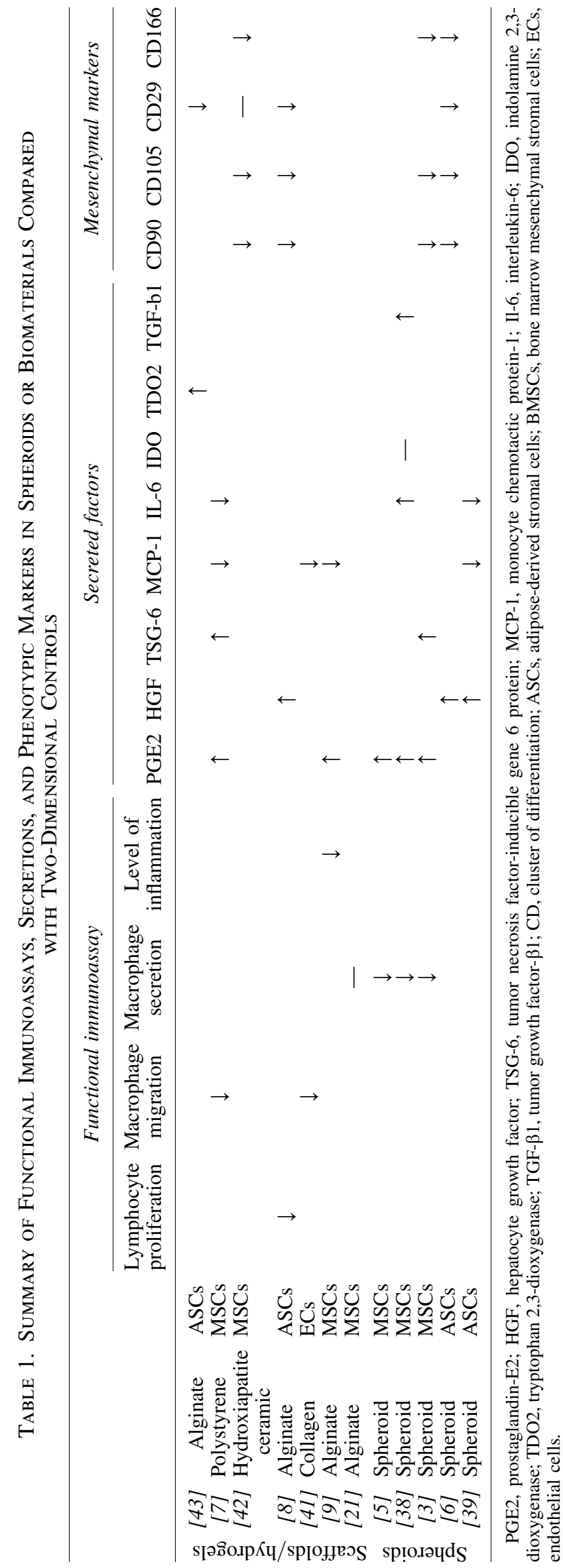


monocyte adhesion more than the conditioned medium from ECs in monolayer. ${ }^{41}$ Although ECs were used instead of MSCs, the study was included in this review for its support to the suggested beneficial effect of 3D culture on the immunosuppressive activity.

MSCs cultured in alginate hydrogels inhibited phytohemagglutinin-stimulated peripheral blood mononuclear cell proliferation more than MSCs from monolayer culture in ${ }^{3} \mathrm{H}$-thymidine incorporation assays. ${ }^{8}$ In addition, TNF- $\alpha$ inflammation in a rat organotypic hippocampal slide culture inflammation model was decreased when cocultured with MSCs in alginate hydrogel compared to coculture with MSCs in 2D. ${ }^{9}$ This was not attributed to the effect of a certain immune cell type.

As with spheroids, PGE2, HGF, and TSG-6 levels have also been found to increase in MSCs seeded in biomaterials. ${ }^{7,8,42}$ It has been observed that the culture of MSCs in hydrogels lead to an increase in PGE2 to a level greater than the level achieved by inflammatory stimulation of a monolayer culture. The inflammatory stimulation did not increase the levels of PGE2 when the MSCs were in hydrogels, which may suggest that the cells had reached their maximum potential for PGE2 upregulation. ${ }^{9}$

Secretion of MCP-1, a macrophage-attractant chemokine, was found to decrease compared to 2D culture in both MSCs in polystyrene and ECs in collagen scaffolds. ${ }^{7,41}$ The decreased secretion correlated with decreased migration and adhesion, respectively. Hence, by decreasing MCP-1 secretion from scaffolds, the MSCs in the scaffold decrease macrophage migration toward the construct, which in turn decrease the potential immunogenic confrontation with macrophages. MCP-1 secretion has been shown not to be decreased in spheroids. ${ }^{39}$ Since spheroids do have the chance of using their limited cell-cell contact, this could be the reason for the differences between the two 3D systems. With biomaterials, there is a chance of immune cell infiltration if sufficient adherence sequences are present. This is not evident with pristine alginate hydrogels, but has been observed with collagen hydrogels. In this study, the pore size was theorized to be the important factor allowing MSC and immune cell interaction. ${ }^{10}$ None of the included studies addressed this with regard to spheroids.

MSC secretion or production of IDO was not investigated in the studies with biomaterials. In MSCs in alginate hydrogels, however, an increase in the gene TDO2, which is related to IDO and performs similar tryptophan catabolism, was found compared to $2 \mathrm{D}$ controls. ${ }^{43}$ This could explain the increased immunomodulation of lymphocyte proliferation also observed using alginate hydrogels. ${ }^{8}$ In contrast to IDO, TDO2 is not induced by inflammatory signals, which means that the increase in TDO 2 could not compensate for lack of IDO. ${ }^{23}$ However, if TDO2 is already increased in MSCs in scaffolds at baseline and IDO is increased when inflammatory signals are presented, the cumulative tryptophan catabolism and associated mechanisms could be greater, leading to more profound effects on the immune cells.

\section{The importance of contact}

Most of the studies included in this review have focused on the paracrine mechanisms, either by using transwells or a conditioned medium. However, it is important to address the consequence of direct cell-cell contact between MSCs and immune cells. Only the surface MSCs in spheroids will be able to establish this contact, and initially almost no contact occurs between MSCs and immune cells in biomaterial culture, due to spatial separation. Therefore, most of the cells in these 3D cultures will not be able to use the contact-dependent suppressive mechanisms, which aid during lymphocyte inhibition, lymphocyte polarization, and DC maturation in cultures with direct contact. ${ }^{24,28,29,44}$ Two studies finding results conflicting with the previously mentioned ones, established direct cell-cell contact between MSCs and immune cells in the 2D controls. In one study, MSCs in alginate hydrogels did not inhibit macrophage secretion more than MSCs with contact in $2 \mathrm{D} .^{21}$ In another study, rabbit MSCs in collagen scaffolds inhibited lymphocyte proliferation to the same level as 2D controls with contact. ${ }^{10}$ Since there was no difference in the two studies, it is apparent that the paracrine effect was enhanced in 3D to compensate for the fact that the MSCs in biomaterials were not able to affect the leukocytes by, for example, CD54 and PD-L1 to the same extent as the MSCs in monolayer.

Cells in 3D have been demonstrated to be more suppressive than cells in 2D, and it appears that for cells in 2D to be as effective as those in 3D, they need the advantage of cell-cell contact. When cocultured in the same culture chamber, MSCs in alginate hydrogel did not inhibit maturation of DCs to the same extent as MSCs from monolayers with contact. ${ }^{8}$ This is in line with observations from other groups who found that inhibition of DC maturation by MSCs was dependent on cellcell contact. $^{24,25}$ These functional data show that even though paracrine immunomodulatory mechanisms are increased in 3D, not all cell types are affected equally, and DCs seem to be more dependent on contact than other immune cells.

\section{The Physical 3D Environment}

It is known that MSCs in spheroids are more secretory than their monolayer counterparts. ${ }^{2}$ It is important to bring to attention that other types of MSC 3D cultures, that is, biomaterials, also produce similar effects. The enhancement may very well be due to different altered functions, but since many of the same secretory factors are upregulated and identical phenotypic changes occur, it is likely that there are shared mechanisms at work. In this study, we will touch upon the differences and potential similarities between the two groups and how they both differ from $2 \mathrm{D}$ culture. The main points are shown in Figure 1.

\section{Three-dimensional strain and rigidity}

The interaction with the surrounding matrix is minimized in spheroids, resulting in the strain by cell-matrix interaction also being minimized. Instead, the strain by cell-cell interaction is increased due to the solidity of the spheroid formation, and this strain is dispersed on all surfaces of the cells with low rigidity (a Young's Modulus of $\sim 60$ Pascal) and not just limited to the basal surface with great rigidity as TCP (Young's Modulus in the range of Gigapascal). ${ }^{45}$ There is more tension on the outer cells in spheroids than the inner cell mass, and mesenchymal cell type as well as method of spheroid assembly also influence this. ${ }^{35}$ This difference in tension creates a certain morphological polarization of the 

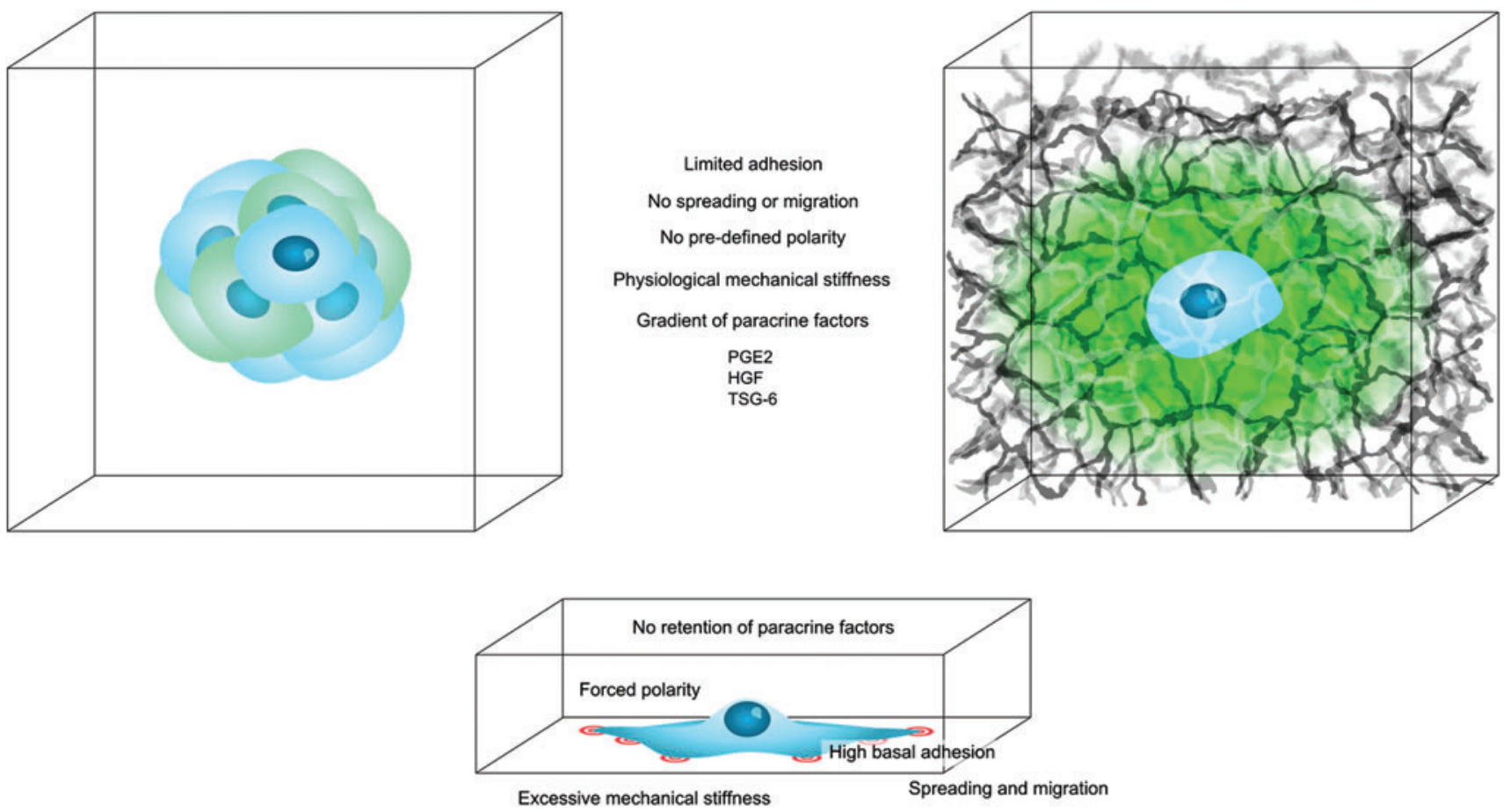

FIG. 1. Overview of the common effects on MSCs by spheroid or biomaterial culture compared to two-dimensional culture. The MSCs in the middle of the spheroids and in the biomaterials, both experience limited adhesion and spreading, lack of polarization, less cellular strain due to less mechanical stiffness of the surroundings, a gradient of paracrine factors, and ultimately a common increase in immunomodulatory mediators PGE2, HGF, and TSG-6. PGE2, prostaglandin-E2; HGF, hepatocyte growth factor; TSG-6, tumor necrosis factor-inducible gene 6 protein; MSC, mesenchymal stromal/stem cell. Color images available online at www.liebertpub.com/teb

cells, although they will not be as polarized as on 2D TCP, since the cells are able to spread in all dimensions. The outer cells are flattened with high expression of integrins, while the inner cells, subjected to less tension, are irregular and display increased levels of cadherins. ${ }^{35}$

There are several similarities between the inner cells in the spheroids and the cells in a biomaterial. For biomaterials, the strain on the mechanosensation apparatus caused by cell-matrix interaction is spread over the entire surface of the cell and not just the basal surface. In addition, the strain is subsidiary, since most biomaterials are softer than 2D TCP, and the cells are able to spread in all directions. In contrast to the outer layers of the spheroids, cells in biomaterials are not restricted to a certain shape, which results in a nonpolarized cell morphology. The tensile strength varies greatly between different biomaterials, even with modifications to the same biomaterial. $^{35,46}$ Adding to this, the rigidity of the different matrices is not constant, since some hydrogels gradually dissolve. ${ }^{8}$

\section{Gas exchange and cytokine concentration gradients}

A mechanism thought to be responsible for many of the beneficial effects of spheroid culture is local hypoxia within the spheroid. ${ }^{35}$ The tight connection between the cells in a confined space creates a local gradient of gas, nutrients, and cytokines. HIF- 1 transcription, which is responsible for many of the beneficial effects with regard to regenerative potential, is primarily regulated by oxygen tension, and also increased in spheroids and thought to be responsible for many of the increased cytokines like HGF. ${ }^{47}$ However, it is also regulated by metabolites under normoxic conditions, and assessing gradients of metabolites and oxygen in different 3D cultures correlated with immunologic abilities remains to be investigated. ${ }^{48}$ In addition, the localization of growth factors has been proposed as a mechanism for increased therapeutic potential of MSC spheroids. ${ }^{35}$ This localization could be due to upregulated extracellular matrix (ECM) secretion in spheroids and explains why ECM retention has been speculated to stimulate autocrine signaling in the spheroids. ${ }^{35,39}$

In contrast to spheroids, no evidence has been found regarding local hypoxia when seeded in hydrogels or other biomaterials. This is most likely due to the lower cell density in these cultures. In addition, the hydrophilicity and porosity of biomaterials enable mass transport to be more efficient compared to spheroids, although gradients could form in culture. As exemplified with the growing embryo, as small as the gradient of nutrients, gasses, and cytokines may be, it has a potential huge impact on the cell culture and cellular fate. ${ }^{1}$ In addition, increases in secretion of ECM from MSCs in biomaterials have also been observed, adding to potentially increased retention of growth factors like in the spheroids. ${ }^{43}$

\section{Adhesion and phenotype}

Interestingly, a decrease in 2D mesenchymal markers as defined by ISCT, ${ }^{12,13}$ was a common observation in $3 \mathrm{D}$ environments. CD105, CD90, CD29, and CD166 were all decreased in spheroids (Table 1). ${ }^{3,6,36}$ Common for all the decreased markers is that they are involved in adhesion. Since 
there are only cells in spheroids, it makes sense that there is a decrease in CD29 since this binds to ECM. The decrease in CD166 and the pericyte marker CD146 is more peculiar due to their association with cellular adhesion. Complicating the matter further, CD90 is also generally involved in cell-cell and cell-matrix interaction, although the exact mechanisms are not fully elucidated. It would seem that the cellular interactions governed by these classical 2D MSC markers are not necessary for the spheroid formation, leading to downregulation.

A similar pattern of decreased expression of 2D mesenchymal markers was evident in biomaterials. ${ }^{8,42,43}$ For biomaterials, the lack of cellular adhesion markers like CD166 is more comprehensive since the cells are more separated. Furthermore, the lack of added ECM to the biomaterials diminishes the need for CD29. Increased CD146, a pericyte marker, was reported by Papadimitropoulos et al., which is most likely not due to cellular interaction or the scaffold itself, but rather the shear flow that was added to the scaffold. $^{42}$

As of now, no direct relationship has been established between the mentioned phenotypic markers and immunomodulation, but the presented evidence does suggest a correlation. The lack of 2D mesenchymal markers does not result in decreased stemness, but may be a return to an in vivo phenotype.

\section{In vivo resemblance}

As described, spheroid cultures were originally used to investigate the spontaneous in vivo spheroid assembly of MSCs trapped in rat lungs. ${ }^{3,49}$ Bartosh et al. showed how expression of the same secreted factors that were increased in vivo became elevated during the first $96 \mathrm{~h}$ of in vitro spheroid assembly, suggesting that the cells behaved in spheroid culture as they would in vivo. ${ }^{4}$ There is evidence that MSCs become more secretory when implanted in vivo, where topography, rigidity, and secretory milieu are different from in vitro conditions. Several groups have found increased secretion of immunoactive and regenerative cytokines in implanted MSCs. ${ }^{3,49,50}$ It should be noted that the included studies all used immune-deficient animals and the effects of transplant on the cells were assessed within a short time period after transplantation. There is evidence that the cells could be affected differently when transplanted to immunocompetent animals or followed for a longer period of time, but this subject is not within the scope of this review. ${ }^{51}$

The downregulation of the classical 2D MSC markers is another example of how both spheroids and biomaterials differ from regular in vitro $2 \mathrm{D}$ TCP culture. It has been shown that these markers increase during the first passages in 2D TCP culture and decrease in both spheroids and biomaterials, which could indicate that the markers are more associated with plastic $2 \mathrm{D}$ adhesion than other physiological functions. ${ }^{36}$

It is possible that not only spheroids but also $3 \mathrm{D}$ environments in general make the cells resemble their native in vivo form more, and the results observed with $3 \mathrm{D}$ culture represent the abilities of the cells in their native environments. Essentially, it may not be the $3 \mathrm{D}$ environment that enhances the immunomodulatory abilities of the MSCs, but the $2 \mathrm{D}$ culture on the rigid TCP that decreases them. This would suggest that the cells lose some of their immunomodulatory potential on $2 \mathrm{D}$, while increasing proliferation and certain phenotypic markers, and this effect is reversed when the cells are reinstated in a 3D environment, largely regardless of variations in this environment.

\section{Conclusion and Perspectives}

MSCs in 3D culture have increased paracrine immunomodulatory potential compared to $2 \mathrm{D}$ culture on TCP, which is evident for both spheroids and biomaterials. This potential seems to be concurrent with downregulation of some 2D mesenchymal markers. Both spheroids and biomaterials present a multitude of extracellular cues and physical conditions like rigidity, gradient of gas, and paracrine mediators and limited spreading, adhesion, and polarity, most of which are not shared between the systems. However, they produce similar effects on the MSCs, with the only overall shared condition that the cells are not in $2 \mathrm{D}$ culture. From an intuitive point of view, it is easy to accept that $3 \mathrm{D}$ culture is closer to normal physiological conditions than 2D, but the exact mechanisms of action remain to be defined. Identification of mutual immunomodulatory enhancing mechanisms behind the mentioned findings would be extremely valuable in terms of priming of cells before therapy and understanding the MSC immunobiology in general.

\section{Limitations}

Because the included studies overall have had other primary outcomes than immunomodulation, correct 2D controls have often been neglected when performing the immunological assays, which diminishes the relevance of such references considerably. Several studies have investigated the combination of MSCs and immune cells when coseeded in $3 \mathrm{D}$ environments. ${ }^{52,53}$ With such experimental designs, it is not possible to isolate the secretions of MSCs from those of immune cells. The included studies all had 2D controls seeded in the same cell density as in the 3D structures. If the experiments have been conducted over several days, there may be an influence by proliferation of the seeded cells. Not all the included studies included measurements of proliferation and therefore could not have corrected for this possibility. Finally, the review is limited by the omnipresent bias of the scientific community in general, exclusively publishing positive results, since there may be several groups who have found no increase in immunosuppressive ability in $3 \mathrm{D}$, but have not published.

\section{Disclosure Statement}

B.F., M.J., A.E.P., J.K., and A.E. have no competing commercial, proprietary, or financial interests. S.C. has a US patent on alginate hydrogel composition.

\section{References}

1. Baker, B.M., and Chen, C.S. Deconstructing the third dimension: how 3D culture microenvironments alter cellular cues. J Cell Sci 125, 3015, 2012.

2. Madrigal, M., Rao, K.S., and Riordan, N.H. A review of therapeutic effects of mesenchymal stem cell secretions and induction of secretory modification by different culture methods. J Transl Med 12, 2602014. 
3. Bartosh, T.J., et al. Aggregation of human mesenchymal stromal cells (MSCs) into 3D spheroids enhances their antiinflammatory properties. Proc Natl Acad Sci U S A 107, 13724, 2010.

4. Bartosh, T.J., Ylöstalo, J.H., Bazhanov, N., Kuhlman, J., and Prockop, D.J. Dynamic compaction of human mesenchymal stem/precursor cells into spheres self-activates caspase-dependent IL1 signaling to enhance secretion of modulators of inflammation and immunity (PGE2, TSG6, and STC1). Stem Cells 31, 2443, 2013.

5. Ylöstalo, J.H., Bartosh, T.J., Coble, K., and Prockop, D. J. Human mesenchymal stem/stromal cells cultured as spheroids are self-activated to produce prostaglandin E2 that directs stimulated macrophages into an anti-inflammatory phenotype. Stem Cells 30, 2283, 2012.

6. Park, I.S., Rhie, J.-W., and Kim, S.-H. A novel threedimensional adipose-derived stem cell cluster for vascular regeneration in ischemic tissue. Cytotherapy 16, 508, 2014.

7. Vallés, G., et al. Topographical cues regulate the crosstalk between MSCs and macrophages. Biomaterials 37, 124, 2015.

8. Follin, B., et al. Human adipose-derived stromal cells in a clinically applicable injectable alginate hydrogel: Phenotypic and immunomodulatory evaluation. Cytotherapy 17, 1104, 2015.

9. Stucky, E.C., Schloss, R.S., Yarmush, M.L., and Shreiber, D.I. Alginate micro-encapsulation of mesenchymal stromal cells enhances modulation of the neuro-inflammatory response. Cytotherapy 17, 1353, 2015.

10. Yuan, T., Li, K., Guo, L., Fan, H., and Zhang, X. Modulation of immunological properties of allogeneic mesenchymal stem cells by collagen scaffolds in cartilage tissue engineering. J Biomed Mater Res A 98, 332, 2011.

11. Hanson, S., D'Souza, R.N., and Hematti, P. Biomaterialmesenchymal stem cell constructs for immunomodulation in composite tissue engineering. Tissue Eng Part A 20, 2162, 2014.

12. Dominici, M., et al. Minimal criteria for defining multipotent mesenchymal stromal cells. The International Society for Cellular Therapy position statement. Cytotherapy 8, 315, 2006.

13. Bourin, P., et al. Stromal cells from the adipose tissuederived stromal vascular fraction and culture expanded adipose tissue-derived stromal/stem cells: a joint statement of the International Federation for Adipose Therapeutics and Science (IFATS) and the International So. Cytotherapy 15, 641, 2013.

14. Yun Cheng, H. The Impact of Mesenchymal Stem Cell Source on Proliferation, Differentiation, Immunomodulation and Therapeutic Efficacy. J Stem Cell Res Ther 4, 237, 2014.

15. Hoogduijn, M.J., et al. The immunomodulatory properties of mesenchymal stem cells and their use for immunotherapy. Int Immunopharmacol 10, 1496, 2010.

16. Caplan, A.I., and Correa, D. The MSC: an injury drugstore. Cell Stem Cell 9, 11, 2011.

17. Le Blanc, K., and Mougiakakos, D. Multipotent mesenchymal stromal cells and the innate immune system. Nat Rev Immunol 12, 383, 2012.

18. Gebler, A., Zabel, O., and Seliger, B. The immunomodulatory capacity of mesenchymal stem cells. Trends Mol Med 18, 128, 2012.

19. Mosser, D.M., and Edwards, J.P. Exploring the full spectrum of macrophage activation. Nat Rev Immunol 8, 958, 2008.
20. Kyurkchiev, D. Secretion of immunoregulatory cytokines by mesenchymal stem cells. World J Stem Cells 6, 552, 2014.

21. Barminko, J., et al. Encapsulated mesenchymal stromal cells for in vivo transplantation. Biotechnol Bioeng 108, 2747, 2011.

22. Grohmann, U. Tolerance, DCs and tryptophan: much ado about IDO. Trends Immunol 24, 242, 2003.

23. Mellor, A.L., and Munn, D. H. IDO expression by dendritic cells: tolerance and tryptophan catabolism. Nat Rev Immunol 4, 762, 2004.

24. Larocca, R.A., et al. Adipose tissue-derived mesenchymal stem cells increase skin allograft survival and inhibit Th-17 immune response. PLoS One 8, e76396 2013.

25. Jiang, X.-X., et al. Human mesenchymal stem cells inhibit differentiation and function of monocyte-derived dendritic cells. Blood 105, 4120, 2005.

26. English, K. Mechanisms of mesenchymal stromal cell immunomodulation. Immunol Cell Biol 91, 19, 2013.

27. Di Nicola, M., et al. Human bone marrow stromal cells suppress T-lymphocyte proliferation induced by cellular or nonspecific mitogenic stimuli. Blood 99, 3838, 2002.

28. Chinnadurai, R., Copland, I.B., Patel, S.R., and Galipeau, J. IDO-independent suppression of T cell effector function by IFN- $\gamma$-licensed human mesenchymal stromal cells. J Immunol 192, 1491, 2014.

29. Ren, G., et al. Inflammatory cytokine-induced intercellular adhesion molecule- 1 and vascular cell adhesion molecule-1 in mesenchymal stem cells are critical for immunosuppression. J Immunol 184, 2321, 2010.

30. Chen, Z., et al. IL-10 and TGF-beta induce alloreactive $\mathrm{CD} 4+\mathrm{CD} 25-\mathrm{T}$ cells to acquire regulatory cell function. Blood 101, 5076, 2003.

31. Melief, S.M., et al. Multipotent stromal cells induce human regulatory $\mathrm{T}$ cells through a novel pathway involving skewing of monocytes toward anti-inflammatory macrophages. Stem Cells 31, 1980, 2013.

32. Sojka, D.K., Huang, Y.-H., and Fowell, D. J. Mechanisms of regulatory T-cell suppression - a diverse arsenal for a moving target. Immunology 124, 13, 2008.

33. Krampera, M., Galipeau, J., Shi, Y., Tarte, K., and Sensebe, L. Immunological characterization of multipotent mesenchymal stromal cells - The International Society for Cellular Therapy (ISCT) working proposal. Cytotherapy 15, 1054, 2013.

34. Bernardo, M.E., and Fibbe, W.E. Mesenchymal stromal cells: sensors and switchers of inflammation. Cell Stem Cell 13, 392, 2013.

35. Sart, S., Tsai, A.-C., Li, Y., and Ma, T. Three-dimensional aggregates of mesenchymal stem cells: cellular mechanisms, biological properties, and applications. Tissue Eng Part B Rev 20, 365, 2014.

36. Frith, J. E., Thomson, B., and Genever, P. G. Dynamic three-dimensional culture methods enhance mesenchymal stem cell properties and increase therapeutic potential. Tissue Eng Part C Methods 16, 735, 2010.

37. Ryan, A.E., et al. Chondrogenic Differentiation increases antidonor immune response to allogeneic mesenchymal stem cell transplantation. Mol Ther 22, 655, 2013.

38. Zimmermann, J.A., and McDevitt, T.C. Pre-conditioning mesenchymal stromal cell spheroids for immunomodulatory paracrine factor secretion. Cytotherapy 16, 331, 2014.

39. Amos, P.J., et al. Human adipose-derived stromal cells accelerate diabetic wound healing: impact of cell formulation and delivery. Tissue Eng Part A 16, 1595, 2010. 
40. Dyer, D.P., et al. TSG-6 inhibits neutrophil migration via direct interaction with the chemokine CXCL8. J Immunol 192, 2177, 2014.

41. Indolfi, L., Baker, A.B., and Edelman, E. R. The role of scaffold microarchitecture in engineering endothelial cell immunomodulation. Biomaterials 33, 7019, 2012.

42. Papadimitropoulos, A., et al. Expansion of human mesenchymal stromal cells from fresh bone marrow in a 3D scaffold-based system under direct perfusion. PLoS One 9, e102359, 2014.

43. Duggal, S., et al. Phenotype and gene expression of human mesenchymal stem cells in alginate scaffolds. Tissue Eng Part A 15, 1763, 2009.

44. Luz-Crawford, P., et al. Mesenchymal stem cells repress Th17 molecular program through the PD-1 pathway. PLoS One 7, e45272, 2012.

45. Baraniak, P.R., et al. Stiffening of human mesenchymal stem cell spheroid microenvironments induced by incorporation of gelatin microparticles. J Mech Behav Biomed Mater 11, 63, 2012.

46. Tsur-Gang, O., et al. The effects of peptide-based modification of alginate on left ventricular remodeling and function after myocardial infarction. Biomaterials 30, 189, 2009.

47. Bhang, S.H., et al. Angiogenesis in ischemic tissue produced by spheroid grafting of human adipose-derived stromal cells. Biomaterials 32, 2734, 2011.

48. Semenza, G.L. HIF-1: upstream and downstream of cancer metabolism. Curr Opin Genet Dev 20, 51, 2010.

49. Lee, R.H., et al. Intravenous hMSCs improve myocardial infarction in mice because cells embolized in lung are activated to secrete the anti-inflammatory protein TSG-6. Cell Stem Cell 5, 54, 2009.
50. Landázuri, N., et al. Alginate microencapsulation of human mesenchymal stem cells as a strategy to enhance paracrinemediated vascular recovery after hindlimb ischaemia. J Tissue Eng Regen Med 2012 [Epub ahead of print]; DOI: 10.1002/term.1680.

51. Ankrum, J.A., Ong, J. F., and Karp, J.M. Mesenchymal stem cells: immune evasive, not immune privileged. Nat Biotechnol 32, 252, 2014.

52. Kaminer-Israeli, Y., Shapiro, J., Cohen, S., and Monsonego, A. Stromal cell-induced immune regulation in a transplantable lymphoid-like cell constructs. Biomaterials 31, 9273, 2010.

53. King, S.N., et al. In vitro characterization of macrophage interaction with mesenchymal stromal cell-hyaluronan hydrogel constructs. J Biomed Mater Res A 102, 890, 2014.

Address correspondence to: Bjarke Follin, MSc

Cardiology Stem Cell Center The Heart Center

Rigshospitalet

Copenhagen University Hospital Juliane Maries Vej 20, Dept. 9302

Copenhagen 2100

Denmark

E-mail: bjarke.follin.larsen@regionh.dk

Received: November 30, 2015

Accepted: February 4, 2016

Online Publication Date: March 10, 2016 